\title{
Nonlinear Flux Approximation Scheme for Burgers Equation Derived from a Local BVP
}

\author{
J. H. M. ten Thije Boonkkamp, N. Kumar, B. Koren, D. A. M. van der Woude, \\ and A. Linke
}

\begin{abstract}
We present a novel flux approximation scheme for the viscous Burgers equation. The numerical flux is computed from a local two-point boundary value problem for the stationary equation and requires the iterative solution of a nonlinear equation depending on the local boundary values and the viscosity. In the inviscid limit the scheme reduces to the Godunov numerical flux.
\end{abstract}

\section{Introduction}

The viscous Burgers equation is a well-known model problem in fluid dynamics, describing the nonlinear convection-diffusion balance for incompressible flow. It was introduced by Burgers in [1] to study turbulence in incompressible flow, and since then it was investigated in many publications.

Solutions of the Burgers equation can exhibit steep interior/boundary layers when the viscosity is small. In the inviscid limit, discontinuous solutions (shock waves) can develop, even when the initial condition is smooth. This puts severe restrictions on the (spatial) discretisation. We pursue to construct a scheme that has the following properties. First, for positive viscosity (bounded below by a positive constant), the scheme should be second order accurate, second, it should not produce spurious oscillations in the vicinity of steep layers, and third, it has a three-point coupling.

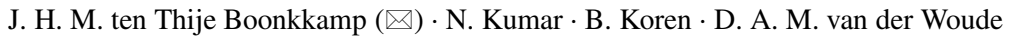
Department of Mathematics and Computer Science, Eindhoven University of Technology, Eindhoven, The Netherlands e-mail: j.h.m.tenthijeboonkkamp@tue.nl; n.kumar@tue.nl; b.koren@tue.nl; d.a.m.v.d.woude@student.tue.nl

A. Linke

Weierstrass Institute for Applied Analysis and Stochastics, Berlin, Germany

e-mail: alexander.linke@wias-berlin.de 
For space discretisation we adopt the finite volume method, hence we need flux approximation schemes. For the inviscid equation, Godunov's flux approximation scheme is very well known. The expression for this flux is derived from a local initial value problem, assuming a piecewise constant initial condition, the so-called Riemann problem. The Godunov scheme is a basic scheme which is only first order accurate. It can be combined with, for example, (W)ENO reconstruction and higher order Runge-Kutta time integration methods to achieve higher order. The viscous term is usually approximated by central differences.

In this contribution we follow a different approach. We compute the numerical flux from a local two-point boundary value problem (BVP) for the steady, viscous Burgers equation, taking into account the fully nonlinear convection-diffusion balance. Our motivation is to derive an extension of the Godunov numerical flux for the viscous equation. This way we anticipate that the numerical solution inherits properties of the exact solution. For example, in Sect. 3 we will show, under certain conditions, that the resulting numerical scheme is monotone, implying that no spurious oscillations are generated. Moreover, we will show that our numerical flux reduces to the Godunov flux for the inviscid equation.

The nonlinear scheme is inspired by the complete flux scheme presented in [6]. The complete flux scheme is a flux approximation scheme for (linear) conservation laws of advection-diffusion-reaction type. The basic idea of the complete flux scheme is to compute the numerical flux from the entire equation, including the source term. Therefore, the numerical flux is the superposition of a homogeneous flux, corresponding to the advection-diffusion operator, and an inhomogeneous flux, taking into account the effect of the source. We like to emphasize that our flux approximation scheme corresponds to the fully nonlinear convection-diffusion operator, and as such can be considered as a modification of the homogeneous flux scheme.

We have organized the paper as follows. In Sect. 2 we present the local BVP defining the numerical flux. Next, in Sect. 3 we give the derivation of the numerical flux. A numerical example is given in Sect. 4 and concluding remarks are given in Sect. 5 .

\section{Local BVP for the Numerical Flux}

In this section we present the fully nonlinear BVP from which we derive the numerical flux (function).

Consider the one-dimensional viscous Burgers equation and corresponding flux $f\left(u, u_{x}\right)$, i.e.,

$$
u_{t}+f\left(u, u_{x}\right)_{x}=0, \quad f\left(u, u_{x}\right)=\frac{1}{2} u^{2}-v u_{x},
$$


where $v \geq 0$ is the viscosity. Equation (1) for $u=u(x, t)$ is defined on, say, $(0,1) \times$ $[0, \infty)$. For space discretisation we employ the finite volume method [2]. Let $\Delta x=$ $1 /(N-1)$ be the grid size, and let $x_{j}=(j-1) \Delta x(j=1,2, \ldots, N)$ and $x_{j+1 / 2}=$ $\frac{1}{2}\left(x_{j}+x_{j+1}\right)(j=1,2, \ldots, N-1)$ denote the grid and interface points, respectively. Integrating (1) over a control volume $\Omega_{j}=\left(x_{j-1 / 2}, x_{j+1 / 2}\right)$, we obtain the semidiscretisation

$$
\Delta x \dot{u}_{j}(t)+F_{j+1 / 2}(t)-F_{j-1 / 2}(t)=0,
$$

where $\dot{u}_{j}(t) \approx u_{t}\left(x_{j}, t\right)$ and $F_{j+1 / 2}(t) \approx f\left(u, u_{x}\right)\left(x_{j+1 / 2}, t\right)$ is the numerical flux at $x=x_{j+1 / 2}$. In the following we suppress the dependency on $t$. The derivation of $F_{j+1 / 2}$ is based on the following local two-point BVP:

$$
\begin{aligned}
& f_{x}=\left(\frac{1}{2} u^{2}-v u_{x}\right)_{x}=0, \quad x_{j}<x<x_{j+1}, \\
& u\left(x_{j}\right)=u_{j}=u_{\mathrm{L}}, \quad u\left(x_{j+1}\right)=u_{j+1}=u_{\mathrm{R}},
\end{aligned}
$$

which we obtain from (1) ignoring the time derivative. In the derivations that follow, it is convenient to normalize the spatial coordinate $x$ in (3). Therefore, we introduce the variables

$$
w(\sigma)=u(x), \quad \sigma=\frac{x-x_{j}}{\Delta x}, \quad \varepsilon=\frac{v}{\Delta x}
$$

and can rewrite the BVP (3) as follows

$$
\begin{aligned}
& \left(\frac{1}{2} w^{2}-\varepsilon w^{\prime}\right)^{\prime}=0, \quad 0<\sigma<1, \\
& w(0)=u_{\mathrm{L}}, \quad w(1)=u_{\mathrm{R}},
\end{aligned}
$$

where the prime $\left(^{\prime}\right)$ denotes differentiation with respect to $\sigma$. From this BVP we will derive expressions for the numerical flux (function) $F_{j+1 / 2}=F\left(u_{\mathrm{L}}, u_{\mathrm{R}} ; \varepsilon\right)$.

Integrating the ODE (5a) twice and applying the boundary conditions (5b) we can derive the following (implicit) representation of the solution [3]

$$
\begin{aligned}
& w(\sigma)=u_{\mathrm{L}}+\left(u_{\mathrm{R}}-u_{\mathrm{L}}\right) \frac{\Lambda(\sigma)}{\Lambda(1)}, \quad w^{\prime}(\sigma)=\left(u_{\mathrm{R}}-u_{\mathrm{L}}\right) \frac{\lambda(\sigma)}{\Lambda(1)}, \\
& \lambda(\sigma)=\exp \left(\frac{1}{\varepsilon} \int_{0}^{\sigma} w(\eta) \mathrm{d} \eta\right)>0, \quad \Lambda(\sigma)=\int_{0}^{\sigma} \lambda(\xi) \mathrm{d} \xi .
\end{aligned}
$$

From this representation it is evident that $w$ is monotone, more precisely, $w$ is monotonically decreasing if $u_{\mathrm{L}}>u_{\mathrm{R}}$ and monotonically increasing if $u_{\mathrm{L}}<u_{\mathrm{R}}$. We will employ this property in the next section. 


\section{Numerical Flux Function}

In this section we derive expressions for the numerical flux from the BVP (5). A similar derivation is given in [5], however, in this contribution we give a different representation of the numerical flux, which provides more insight. Further, we include a brief discussion on monotonicity and order of convergence.

Because of the monotonicity property of $w$, we distinguish the two cases $u_{\mathrm{L}} \geq$ $u_{\mathrm{R}}$ and $u_{\mathrm{L}}<u_{\mathrm{R}}$.

\subsection{The Case $u_{\mathrm{L}} \geq u_{\mathrm{R}}$}

The solution of BVP (5) satisfies $w^{\prime}(\sigma) \leq 0$, hence $w$ is monotonically decreasing. We define the numerical flux $F_{j+1 / 2}$ by the relation

$$
F_{j+1 / 2}=\frac{1}{2} w^{2}-\varepsilon w^{\prime}=\frac{1}{2} c^{2}>0,
$$

where $w$ is the solution of BVP (5). Obviously, $F_{j+1 / 2} \geq 0$. Therefore, we can write $F_{j+1 / 2}=\frac{1}{2} c^{2}$ for some unknown $c$ satisfying $|c|>c^{+}=\max \left(\left|u_{\mathrm{L}}\right|,\left|u_{\mathrm{R}}\right|\right)$. The latter inequality readily follows if we substitute $\sigma=0$ or $\sigma=1$ in (7). Relation (7) is equivalent to the following first order ODE for $w$ :

$$
\frac{\mathrm{d} w}{\mathrm{~d} \sigma}=\frac{1}{2 \varepsilon}\left(w^{2}-c^{2}\right) .
$$

Integrating (8) across $(0,1)$ and applying the boundary conditions $(5 \mathrm{~b})$ we find for $c$ the algebraic relation

$$
H^{+}(c)=\log \left(\frac{1+z(c)}{1-z(c)}\right)=\frac{c}{\varepsilon}, \quad z(c)=\frac{\left(u_{\mathrm{L}}-u_{\mathrm{R}}\right) c}{c^{2}-u_{\mathrm{L}} u_{\mathrm{R}}},
$$

where $0<z(c)<1$; see Fig. $1 . H^{+}(c)$ and $c / \varepsilon$ are odd functions of $c$, consequently Eq. (9) has the trivial root $c=0$, which we discard. Since $F_{j+1 / 2}=\frac{1}{2} c^{2}$ is even, we restrict ourselves to $c>c^{+}$. We can prove that $H^{+}(c)=c / \varepsilon$ has a unique solution $c>c^{+}$, which we compute by Newton iteration. If we choose an initial guess $c_{0}>c^{+}$the iteration will always converge.

Note that Eq. (9) implicitly defines $c=c\left(u_{\mathrm{L}}, u_{\mathrm{R}}\right)$. Invoking the implicit function theorem, we conclude that $\partial c / \partial u_{\mathrm{L}}>0$ and $\partial c / \partial u_{\mathrm{R}}<0$ for $c>c^{+}$, implying that

$$
\frac{\partial F_{j+1 / 2}}{\partial u_{\mathrm{L}}}>0, \quad \frac{\partial F_{j+1 / 2}}{\partial u_{\mathrm{R}}}<0 .
$$




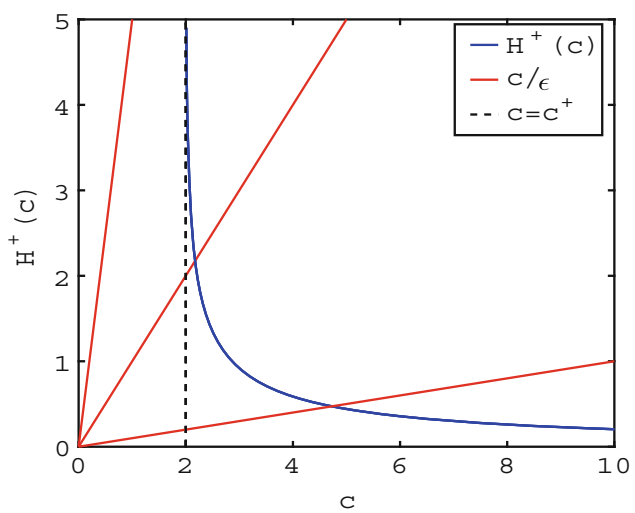

Fig. 1 Intersection points of the function $H^{+}(c)$ with $c / \varepsilon$, restricted to $c>c^{+}$. Parameter values are: $u_{\mathrm{L}}=2, u_{\mathrm{R}}=1$ and $\varepsilon=0.2,1,10$
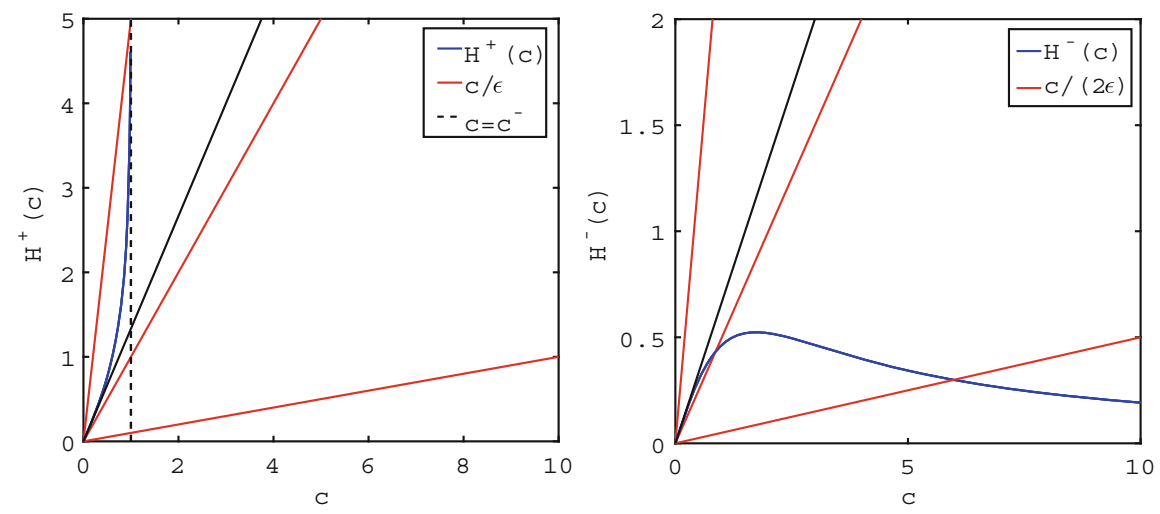

Fig. 2 Intersection points of $H^{+}(c)$ and $c / \varepsilon$ (left), restricted to $0<c<c^{-}$, and $H^{-}(c)$ and $c /(2 \varepsilon)$ (right). Parameter values are: $u_{\mathrm{L}}=1, u_{\mathrm{R}}=3$ and $\varepsilon=0.2,1,10$

When combined with the explicit Euler time integration method, with a time step small enough to satisfy the stability condition, the resulting generalization of Godunov's scheme remains monotone.

\subsection{The Case $u_{\mathrm{L}}<u_{\mathrm{R}}$}

In this case $w^{\prime}(\sigma)>0$ and $w$ is monotonically increasing. Again, the numerical flux is defined by $F_{j+1 / 2}=\frac{1}{2} w^{2}-\varepsilon w^{\prime}$ with $w$ the solution of BVP (5), however, this time we either have $F_{j+1 / 2} \geq 0$ or $F_{j+1 / 2}<0$. For a positive numerical flux we have $F_{j+1 / 2}=\frac{1}{2} c^{2}$ where $c$ is the solution of (9) satisfying $|c|<c^{-}=$ $\min \left(\left|u_{\mathrm{L}}\right|,\left|u_{\mathrm{R}}\right|\right)$; see Fig. 2. This inequality is again a consequence of Eq. (7) if we 
substitute $\sigma=0$ and $\sigma=1$. On the other hand, for a negative flux we have

$$
F_{j+1 / 2}=\frac{1}{2} w^{2}-\varepsilon w^{\prime}=-\frac{1}{2} c^{2}<0,
$$

for the unknown $c$. From (10) we can readily derive the ODE:

$$
\frac{\mathrm{d} w}{\mathrm{~d} \sigma}=\frac{1}{2 \varepsilon}\left(w^{2}+c^{2}\right) .
$$

Integrating this equation across $(0,1)$ and applying the boundary conditions $(5 \mathrm{~b})$ we obtain the relation

$$
H^{-}(c)=\arctan \left(\frac{c}{u_{\mathrm{L}}}\right)-\arctan \left(\frac{c}{u_{\mathrm{R}}}\right)+\frac{\pi}{2}\left(\operatorname{sgn}\left(u_{\mathrm{R}}\right)-\operatorname{sgn}\left(u_{\mathrm{L}}\right)\right)=\frac{c}{2 \varepsilon},
$$

see Fig. 2. In the derivation of (12) we used the identity $\arctan (z)+\arctan (1 / z)=$ $\frac{\pi}{2} \operatorname{sgn}(z)$ for $z \neq 0$. Analogous to the previous case, $H^{-}(c)$ and $c /(2 \varepsilon)$ are odd and $F_{j+1 / 2}=-\frac{1}{2} c^{2}$ is even, therefore we restrict ourselves to $c>0$. For positive $c$ Eq. (12) has a unique solution, which we compute with Newton iteration.

\subsection{Choice of the Numerical Flux}

In the case $u_{\mathrm{L}}<u_{\mathrm{R}}$ we can have either a positive or a negative flux, so we have to determine its sign. To that purpose we investigate the zero-flux condition, i.e., we solve Eq. (7) for $c=0$ subject to the boundary conditions (5b). This way we find the condition

$$
\frac{1}{u_{\mathrm{L}}}-\frac{1}{u_{\mathrm{R}}}=\frac{1}{2 \varepsilon}
$$

In [4] it is proven that, in case $u_{\mathrm{L}} u_{\mathrm{R}}>0$, the numerical flux $F_{j+1 / 2}>0$ for $1 / u_{\mathrm{L}}-1 / u_{\mathrm{R}}<1 /(2 \varepsilon)$, and $F_{j+1 / 2}<0$ for $1 / u_{\mathrm{L}}-1 / u_{\mathrm{R}}>1 /(2 \varepsilon)$. This situation is displayed in Fig. 2. For $\varepsilon=0.2$ Eq. (9) has a unique root $0<c<c^{-}$and consequently $F_{j+1 / 2}>0$. In the other two cases Eq. (12) has a unique solution $c>0$ and $F_{j+1 / 2}<0$. The black lines are tangent to either $H^{+}(c)$ or $H^{-}(c)$ at $c=0$ and correspond to the zero-flux condition (13). Otherwise, for $u_{\mathrm{L}} u_{\mathrm{R}}<0$, the numerical flux $F_{j+1 / 2}<0$. This situation is shown in Fig. 3. Clearly, in this case only Eq. (12) has a solution.

Putting everything together, we obtain the following expressions for the numerical flux:

- if $u_{\mathrm{L}} \geq u_{\mathrm{R}}$

$$
F_{j+1 / 2}=\frac{1}{2} c^{2}, \quad H^{+}(c)=\frac{c}{\varepsilon} \quad\left(c>c^{+}\right) .
$$



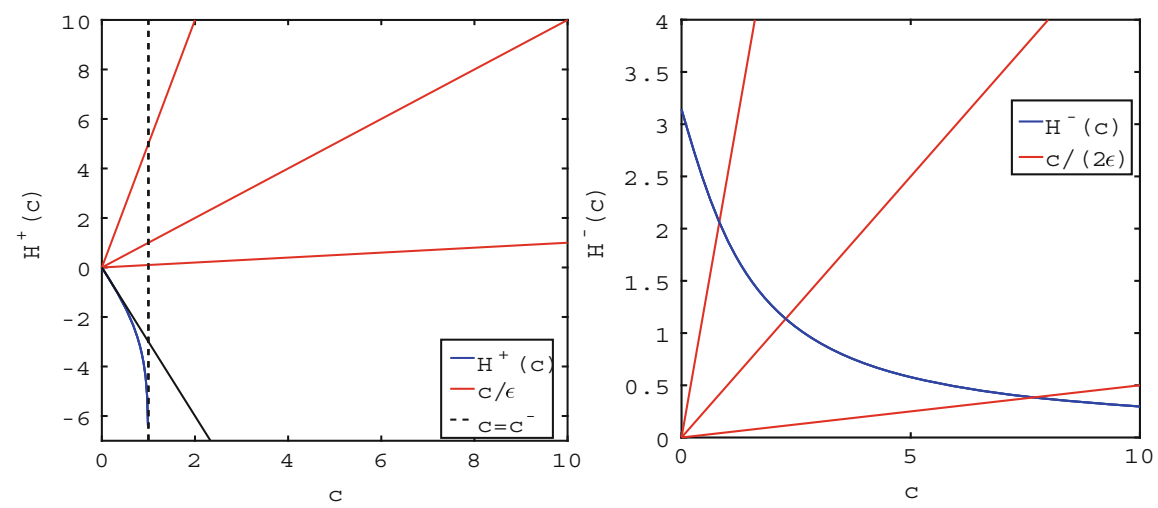

Fig. 3 (Possible) intersection points of $H^{+}(c)$ and $c / \varepsilon$ (left) and $H^{-}(c)$ and $c /(2 \varepsilon)$ (right). Parameter values are: $u_{\mathrm{L}}=-2, u_{\mathrm{R}}=1$ and $\varepsilon=0.2,1,10$

- if $u_{\mathrm{L}}<u_{\mathrm{R}}$ and $u_{\mathrm{L}} u_{\mathrm{R}}>0$

$$
F_{j+1 / 2}=\left\{\begin{array}{cll}
-\frac{1}{2} c^{2} & \text { if } \quad \frac{1}{u_{\mathrm{L}}}-\frac{1}{u_{\mathrm{R}}}>\frac{1}{2 \varepsilon}, & H^{-}(c)=\frac{c}{2 \varepsilon} \\
\frac{1}{2} c^{2} & \text { if } \quad \frac{1}{u_{\mathrm{L}}}-\frac{1}{u_{\mathrm{R}}}<\frac{1}{2 \varepsilon}, & H^{+}(c)=\frac{c}{\varepsilon} \quad\left(0<c<c^{-}\right), \\
0 & \text { if } \quad \frac{1}{u_{\mathrm{L}}}-\frac{1}{u_{\mathrm{R}}}=\frac{1}{2 \varepsilon}
\end{array}\right.
$$

- if $u_{\mathrm{L}}<u_{\mathrm{R}}$ and $u_{\mathrm{L}} u_{\mathrm{R}}<0$

$$
F_{j+1 / 2}=-\frac{1}{2} c^{2}, \quad H^{-}(c)=\frac{c}{2 \varepsilon} .
$$

The sign of the numerical flux as a function of $u_{\mathrm{L}}$ and $u_{\mathrm{R}}$ is displayed in Fig. 4.

To conclude, we consider the convective and diffusive limits. First, for pure convection $(v=0)$ the numerical flux reduces to the Godunov numerical flux. From Fig. 4 we observe that for decreasing $\varepsilon$, and hence decreasing $v$, the region where $F_{j+1 / 2}<0$ recedes to the quadrant where $u_{\mathrm{L}}<0$ and $u_{\mathrm{R}}>0$. Note however, that in the limit $v=0$ Eq. (12) has the solution $c=0$, giving $F_{j+1 / 2}=0$, which is in agreement with Godunov's flux. Second, we consider the diffusive limit $(v \rightarrow \infty)$, restricting ourselves to $u_{\mathrm{L}} \geq u_{\mathrm{R}}$. Note that $z(c) \rightarrow 0$ for $v \rightarrow \infty$. Applying the approximation $H^{+}(c) \approx 2 z(c)$ for $|z(c)| \ll 1$, we obtain from Eq. (9) that $c^{2}-u_{\mathrm{L}} u_{\mathrm{R}} \approx 2 \varepsilon\left(u_{\mathrm{L}}-u_{\mathrm{R}}\right)$. For the numerical flux we then find

$$
F_{j+1 / 2} \approx \frac{1}{2} u_{j} u_{j+1}-\frac{v}{\Delta x}\left(u_{j+1}-u_{j}\right),
$$




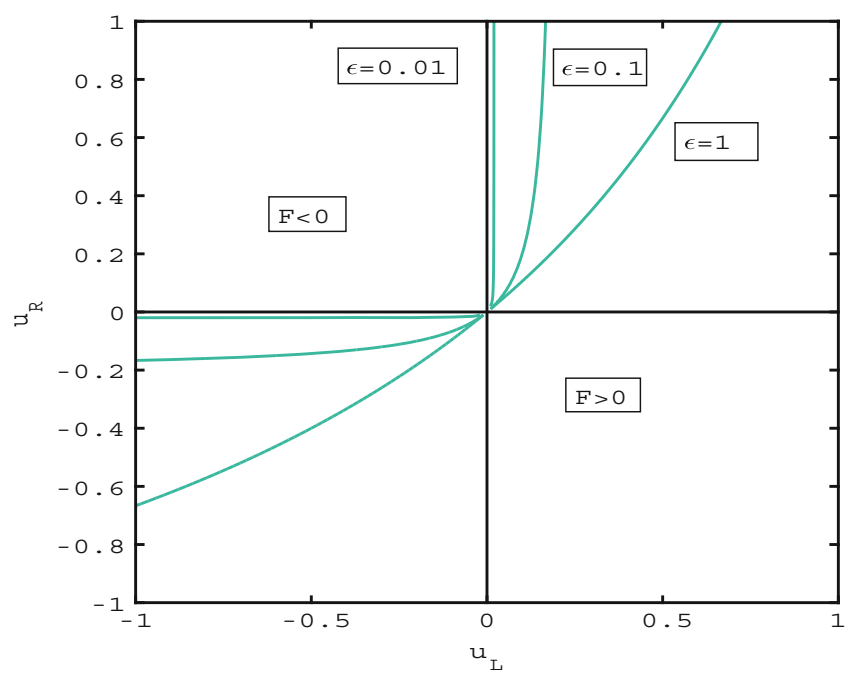

Fig. 4 Sign of the numerical flux function. The contours denote the zero-flux condition for several values of $\varepsilon$

which is a second order accurate approximation of $f\left(u, u_{x}\right)$. These limit cases are in agreement with the observation that the scheme exhibits first order convergence for small $\varepsilon$, gradually increasing to second order for larger $\varepsilon$ [5].

\section{Numerical Example}

In this section we present an example of our scheme; convergence tests have been reported in [5].

We apply our numerical scheme to Eq. (1) subject to the initial condition $u(x, 0)=1+\sin (2 \pi x)$ for $0<x<1$. We compute numerical solutions for $v=10^{-3}$. The initial smooth profile steepens and a typical solution is displayed in Fig. 5. We compute the numerical solutions for $\Delta x=1 / 20,1 / 80,1 / 320$ and compare these with a very accurate reference solution. For time integration we use a third order stability preserving Runge-Kutta method with time step $\Delta t=10^{-5}$, hence the temporal discretisation error is negligible. Newton iteration for Eq. (9) or (12) is terminated if both the residual and the update (in absolute value) drop below the tolerance value $10^{-12}$. Clearly, the steep layer is nicely approximated for decreasing grid size. 


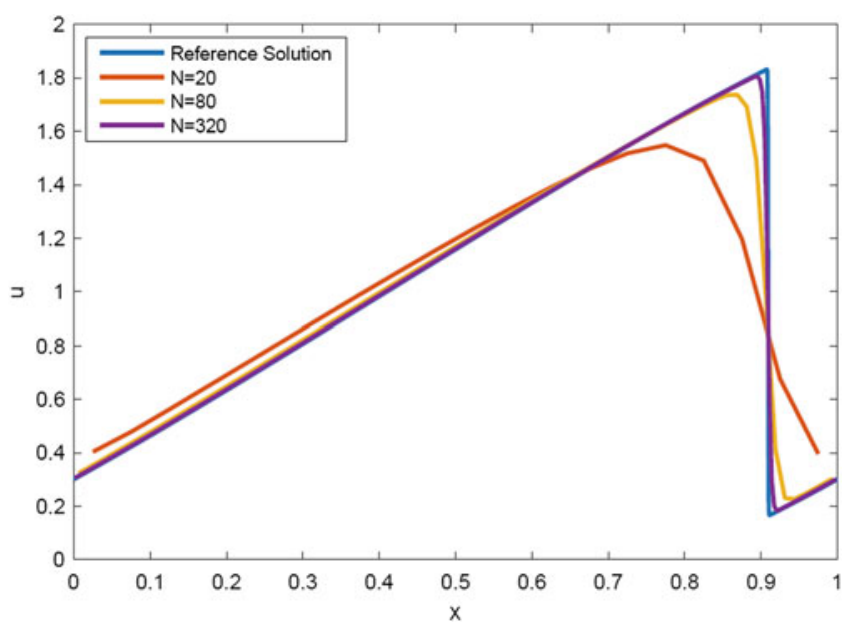

Fig. 5 Snapshot of a numerical solution of the Burgers equation at $t=0.41$

\section{Concluding Remarks}

We have presented a novel flux approximation scheme for the viscous Burgers equation, derived from a local two-point boundary value problem for the stationary equation. The scheme is fully nonlinear, and requires the iterative solution of a nonlinear equation, for which we use Newton iteration. In the limit of zero viscosity, the numerical flux reduces to the Godunov flux. First numerical results are encouraging, however, more numerical tests are required.

As possible extensions, we mention, first, inclusion of the time derivative in the flux approximation, and second, extension to two-dimensional equations.

\section{References}

1. J.M. Burgers, A Mathematical Model Illustrating the Theory of Turbulence (Academic Press, New York, 1948)

2. R. Eymard, T. Gallouët, R. Herbin, Finite volume methods, in Handbook of Numerical Analysis, ed. by P.G. Ciarlet, J.L. Lions, vol. VII (North-Holland, Amsterdam, 2000), pp. 713-1020

3. R. Eymard, J. Fuhrmann, K. Gärtner, A finite volume scheme for nonlinear parabolic equations derived from one-dimensional local Dirichlet problems. Numer. Math. 102, $463-495$ (2006)

4. N. Kumar, Flux approximation schemes for flow problems using local boundary value problems, PhD Thesis, Eindhoven University of Technology, 2017

5. N. Kumar, J.H.M. ten Thije Boonkkamp, B. Koren, A. Linke, A nonlinear flux approximation scheme for the viscous Burgers equation, in Finite Volumes for Complex Applications VIII - Hyperbolic, Elliptic and Parabolic Problems, ed. by C. Cances, P. Omnes (Springer, Switzerland, 2017), pp. 457-465

6. J.H.M. ten Thije Boonkkamp, M.J.H. Anthonissen, The finite volume-complete flux scheme for advection-diffusion-reaction equations. J. Sci. Comput. 46, 47-70 (2011) 